\title{
Culture and Sensitivity Pattern of Urinary Tract Infection in Hospitalized Children in Patan Hospital
}

\author{
Amatya $\mathrm{P}^{1}$, Joshi $\mathrm{S}^{2}$, Shrestha $\mathrm{S}^{3}$
}

${ }^{1}$ Dr. Puja Amatya, MBBS, MD, Lecturer, ${ }^{2} \mathrm{Dr}$. Suchita Joshi, MBBS. MD, Assistant Professor, ${ }^{3}$ Dr. Shrijana Shrestha, MBBS. MD. Professor. All from the department of Paediatrics, Patan Academy of Health Sciences.

\section{Address for correspondence:}

Dr. Puja Amatya, Lecturer

Patan Academy of Health Sciences (PAHS)

Lagankhel, Lalitpur, Nepal

Tel: +9779841401394

E-mail: pujaamatya@pahs.edu.np

Acknowledgements: Mr. Krishna Prajapati, Department of Microbiology, Patan hospital Funding: Nil

Conflict of Interest: None

Permission from IRB: Yes

Ethical dilemmas faced during study: None

\section{How to cite}

Amatya $\mathrm{P}$, Joshi $\mathrm{S}$, Shrestha S. Culture and Sensitivity Pattern of Urinary Tract Infection in Hospitalized Children in Patan Hospital. J Nepal Paediatr Soc 2016;36(1):28-33.

doi: http://dx.doi.org/10.3126/jnps.v36i1.14629

This work is licensed under a Creative Commons Attribution 3.0 License.

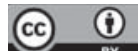

\begin{abstract}
Introduction: Urinary tract infection is one of the most common infections in children. Antibiotics are usually given empirically before urine culture reports are available. The primary aim of this study was to see the causative organisms and culture and sensitivity pattern of urinary tract infection in hospitalized children. Material and Methods: All children aged 0-15 years with culture positive urinary tract infections who were admitted to children's ward from $14^{\text {th }}$ April 2013 to $14^{\text {th }}$ April 2014 were included. The causative organisms for urinary tract infection along with its antibiotic sensitivity pattern were retrospectively reviewed and analyzed. Results: 48 cases of culture positive urinary tract infection were enrolled in this study in a period of 12 months. The most common causative organism was Escherichia coli $(67 \%)$, followed by Klebsiella pneumoniae (21\%), Non-hemolytics streptococcus (4\%), Enterobacter (2\%), Acinetobacter (2\%), Proteus (2\%) and Coagulase negative staphylococcus (2\%). Most cases of the culture positive urinary tract infection occurred between 2 months to 1 year of age. Out of 48 cases, $90 \%$ were sensitive to Amikacin and $85 \%$ were resistant to Ofloxacin. Conclusion: Escherichia coli is the most common organism causing urinary tract infection in children. As resistant to first line antibiotic is increasing, antibiotic stewardship programme should be strengthened.
\end{abstract}

Key words: Urinary tract infection (UTI), Antibiotics

\section{Introduction}

U rinary tract infection (UTI) is one of the most common infections in children. Antibiotics are usually given empirically before the laboratory results of urine culture are available. To ensure appropriate therapy, current knowledge of the organisms that cause UTI and their antibiotic susceptibility is mandatory ${ }^{1}$. The spectrum of etiologic agents causing urinary tract infection and their antimicrobial resistance pattern has been continuously changing over the years, both in community and in hospitals ${ }^{2}$. Escherichia coli is still the most frequently isolated pathogen followed by Klebsiella spp, Proteus, and Enterobacter $s p p^{3,4}$. Especially, in the underdeveloped and developing countries, 
due to inappropriate use of antibiotics, antibiotic resistance is increasing and treatment of urinary tract infection is becoming more difficult. Physicians should understand the regional antibiotic resistance pattern for determining empirical therapy until the results for culture and sensitivity are available. The knowledge on local antibiotic susceptibility is not only helpful for empirical therapy but also necessary to cycle the antibiotics regularly.

According to the hospital policy and guideline, the first line antibiotic used for UTI in children in our hospital is Ofloxacin. Anecdotally, it was noted that children treated with oral Ofloxacin in the outpatient department were increasingly being admitted due to non-response to treatment. Therefore, the objectives of this study were to investigate the common microorganisms causing urinary tract infections and to identify the status of their antibiotic susceptibility in those children who were admitted with UTI.

\section{Material and Methods}

This retrospective study was performed on patients admitted to the children's ward of Patan hospital with a diagnosis of culture positive UTI. All children aged 0-15 years with culture positive UTI who were admitted to children's ward from 14 $4^{\text {th }}$ April 2013 to $14^{\text {th }}$ April 2014 were included. The clinical as well as demographic data of each case was reviewed and recorded in the patient proforma. The case notes were reviewed along with investigation reports to identify the most common pathogens causing urinary tract infection as well as the sensitivity pattern.

Urine samples were collected according to Paediatric protocol of Patan hospital. Catheter sample was collected in children with no urinary control and midstream clean catch collection in children with urinary control.

\section{Criteria for culture positive UTI ${ }^{5}$}

\begin{tabular}{cc}
\hline Method of collection & Colony count \\
\hline Suprapubic aspiration & $\begin{array}{c}\text { Urinary pathogen in any } \\
\text { number }\end{array}$ \\
\hline Urethral catheterization & $>=50 \times 10^{3} \mathrm{CFU} / \mathrm{ml}$ \\
\hline Midstream clean catch & $>10^{5} \mathrm{CFU} / \mathrm{ml}$ \\
\hline
\end{tabular}

CFU: colony forming units

\section{Bacterial isolates and antimicrobial testing}

A total 48 bacterial isolates were identified from 48 patients. Urine microscopy was sent in all cases along with culture and sensitivity. These isolates were identified in microbiology laboratory of Patan hospital. Urine samples were plated out on selective agar plates.

Antimicrobial susceptibilities were performed locally using Kirby disc diffusion method. In this method urine sample was collected in urine culture bottle. 1 micro litre (calibrated loop) of urine sample was inoculated on Blood and MacConkey agar and the plates were incubated at $37 \mathrm{C}$ for 24 hours. If there was growth of bacteria, the colony forming unit( CFU) was counted. And if no growth, no growth after 24 hours was reported. For Gram negative bacilli, the biochemical identification was done by Motility Indole Urea (MIU), Triple Sugar Iron (TSI) and Citrate whereas for Gram positive cocci, Catalase and Coagulase test was done. The Antibiotic Susceptibility Test was done on Muiller Hilton Agar (MHA) in which MHA plate was incubated for 16 to 18 hours and the zone of inhibition was measured. The results were recorded as susceptible, resistant and intermediate according to Clinical Laboratory Standard Institute Antimicrobial susceptibility testing guidelines.

Antibiotics tested as first line were Penicillin/ Amoxycillin, Erythromycin, Oxacillin, Cefotaxim, Cotrimoxazole, Nitrofurantoin, Nalidixic acid, Ciprofloxacin Ofloxacin, Amikacin, Gentamicin. The second line antibiotics tested were Chloramphenicol, Piperacillin-Tazobactum, Imipenem, Meropenem, Colistin and Tigecyclin,

The data were recorded and analyzed using Microsoft excel 2010.

The protocol for this article and ethical approval form for IRB was submitted to the IRC of Patan hospital and approval taken.

\section{Results}

There were forty eight patients with culture positive UTI admitted in children's ward from $14^{\text {th }}$ April 2013 to $14^{\text {th }}$ April 2014. The number of patients admitted with UTI according to the age group distribution is shown in Fig 1.

According to the seasonal variation, there were two peaks of increased admission of patients with UTI were noted. First peak was noticed on Bhadra (August/ September) in which 11 cases of culture positive UTI were admitted followed by second peak in Mangsir (November/December) in which nine cases were admitted. UTI was more common in summer (50\%) followed by winter $(23 \%)$, autumn $(17.5 \%)$ and spring $(17.5 \%)$ respectively. 
Out of 48 patients, $80 \%(\mathrm{~N}=38)$ had fever at the time of presentation. Those who didn't had fever at presentation were all less than two months of age. Other common symptoms at admission were vomiting, pain or crying during micturition and loose stool. Three patients had neonatal jaundice. Among 48 admitted culture positive UTI cases, three cases had one episode and one had two previous episodes of UTI. None of the patients had congenital abnormality of urinary tract like vesico-ureteric reflux. Out of forty eight patients, three also had pyelonephritis on ultrasonography. One had bilateral calcification of renal pelvicalyceal system, two had echogenic kidneys and one had mild hydronephrosis on ultrasonography.

The most common bacteria isolated in urine culture was Escherichia coli $67 \%(\mathrm{~N}=32)$ followed by Klebsiella pneumoniae $21 \%(\mathrm{~N}=10)$. The pattern of bacteria causing UTI is shown in Figure 2.

Out of thirty two Escherichia coli positive cases, 30 cases (93\%) were resistant to Amoxycillin followed by Nalidixic acid in $28(87.5 \%)$ cases, Ciprofloxacin in $27(84.3 \%)$ cases, Cefotaxim in $26(81.2 \%)$ cases, Ofloxacin in $26(81.2 \%)$ cases, Cotrimoxazole in 22 $(68.75 \%)$ cases, Getamicin in $17(53.1 \%)$ cases, Nitrofurantoin in $15(46.8 \%)$ cases and Amikacin in 5 $(15.6 \%)$ cases. Fig 3.

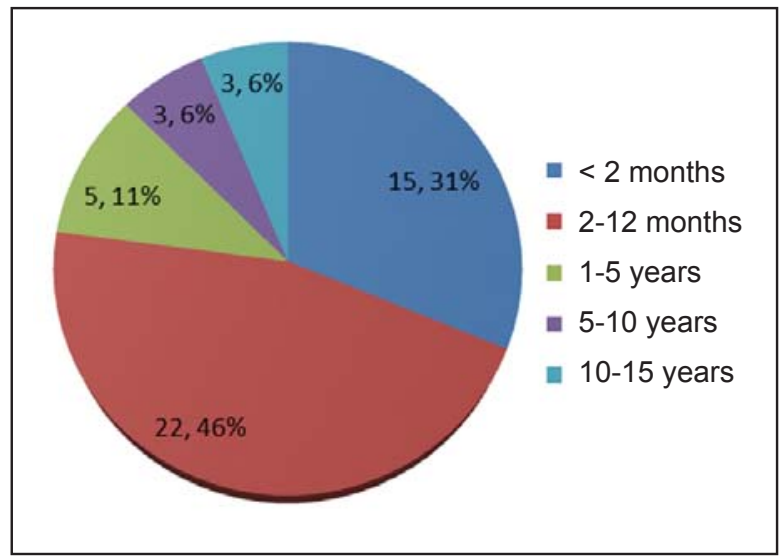

Fig 1: Depicting age wise distribution of cases of the study population.
Out of eleven case of Klebsiella pneumoniae, Amoxycillin was resitant in $10(100 \%)$ cases followed by Nitrofurantoin in $8(80 \%)$ cases, Gentamicin in 7 (70\%) cases, Cotrimoxazole in $7(70 \%)$ cases, Cefotaxim in $7(70 \%)$ cases, Cefotaxim in $7(70 \%)$ cases, Nalidixic acid in $7(70 \%)$ cases, Ciprofloxacin in $6(60 \%)$ cases, Ofloxacin in $4(40 \%)$ cases and Amikacin in $2(20 \%)$ cases. Fig 4.

In this study, the Enterobacter was sensitive only to Ofloxacin and Colistin. Non-hemolytic streptococcus was sensitive only to Amoxicillin and Chloramphenicol. Coagulase negatives staphylococcus aureus was sensitive to Oxacillin, Erythromycin and Ofloxacin. Acinetobacter was sensitive to only to Amikacin.

Out of forty eight urine culture positive cases three organisms were resistant to all first line antibiotics. Out of three, two were Klebsiella pneumoniae and one was Escherichia coli. Both were sensitive only to Meropenem, Imipenem and Colistin .

Among all the patients, 58\% received Amikacin followed by Ofloxacin, Cefotaxim, Chloramphenicol, Vancomycin and Meropenem in 17\%, 15\%, 6\%, 2\%, 2\% respectively.

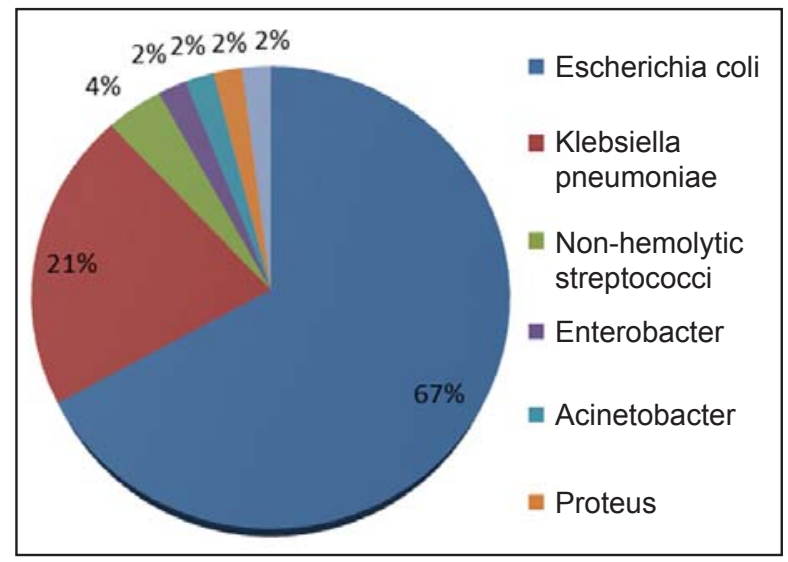

Fig 2: Showing Common pathogens causing urinary tract infections during the study. 


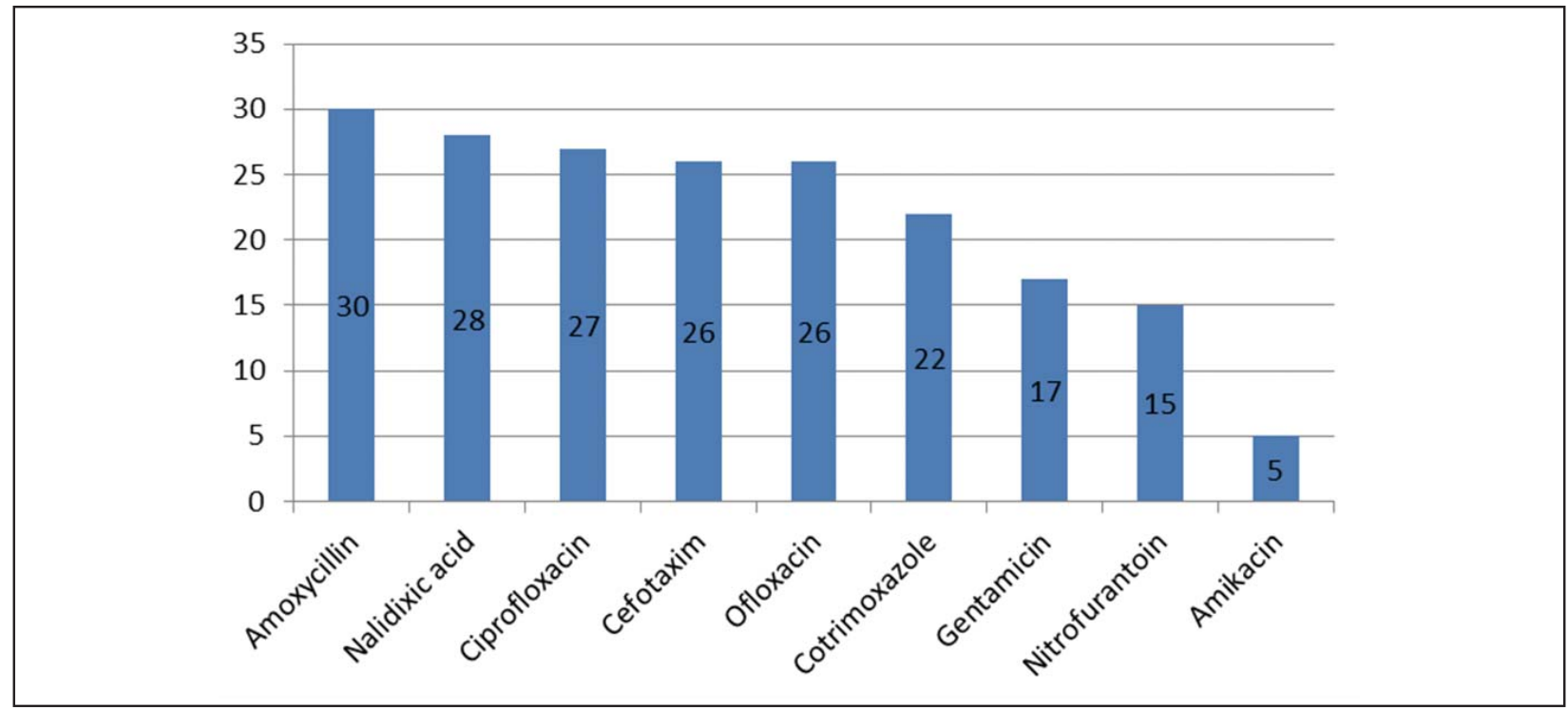

Fig 3: Culture and Sensitivity of Escherichia coli $(n=32)$ in the study.

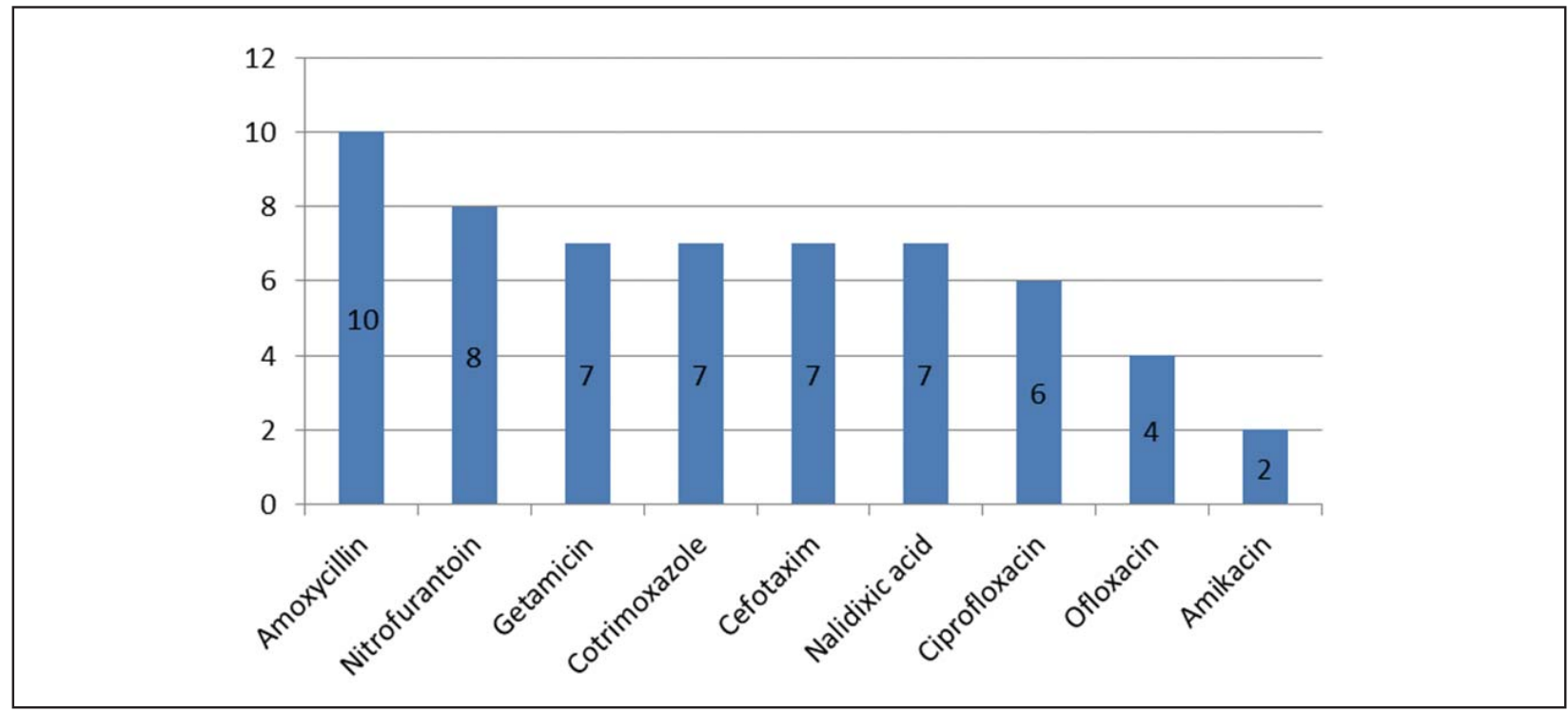

Fig 4: Culture and Sensitivity of Klebsiella pneumoniae $(n=10)$ in the study.

\section{Discussion}

Escherichia coli is the most commonly reported pathogen causing $\mathrm{UTT}^{6,7,8,9,10}$. This is also reflected in this study.

In the study done by Durgesh et $\mathrm{al}^{7}$, Escherichia coli was the predominant $(31.25 \%)$ isolates causing UTI, followed by Staphylococcus aureus (25\%), Pseudomonas aeruginosa (15.62\%), Proteus mirabilis (15.62\%), Klebsiella pneumoniae (6.25\%) and Serratia marcescens $(6.25 \%)$ where as in our study the most common organism causing UTI was Escherichia coli (67\%) followed by Klebsiella pneumoniae (21\%), Non haemolytic streptococcus (4\%), Enterobacter (2\%), Acinetobacter (2\%), Proteus (2\%) and Coagulase negative staphylococcus aureus (2\%). Similarly, in the study done by Yolbaset $\mathrm{al}^{6}$ and Mostafa et $\mathrm{al}^{8}$ and, Escherichia coli was the most common organism causing urinary tract infection followed by Klebsiella which was similar to our study. Similar results were observed in studies done by Rehamanet $\mathrm{al}^{9}$ and $\mathrm{CW}$ kwan et $a^{10}$. This difference may be due to the variation in geographical distribution.

Study done by Haller et al showed the effective empirical intravenous and oral antibiotics for the treatment of community-acquired UTIs include Ampicillin and Aminoglycosides ${ }^{11}$, whereas the oral antibiotic of choice by Prais $D$ et $\mathrm{al}^{12}$ and Hoberman et $\mathrm{al}^{13}$ showed Amoxicillin-Clavulanate or Cefuroxime and Cefixime respectively. In our study, uropathogens showed 
increased resistant pattern to oral antibiotics (Fig 3,4). The reason may be easy availability to antibiotics from pharmacy. We haven't seen the sensitivity with oral Cefixime as this disc was not available while this study was done. Hence this study cannot recommend the choice of empirical oral antibiotic.

Compared to the study done by Yolbas et $\mathrm{al}^{6}$, in which Escherichia coli was resistant to Amikacin in 3\%, Nitrofurantoin 9\%, Trimethoprim/ Sulfamethoxazole $58 \%$ and Cefotaxime $51 \%$, in our study Escherichia coli showed more resistant pattern to these antibiotics i.e Amikacin (15.6\%), Nitrofurantoin (46.8\%), Cotrimoxazole (68.75\%) and Cefotaxim (81\%). The reason for increase in resistance may be due to the excessive use of third generation Cephalosporins both as oral and intravenous route.

In the study done by Durgesh et $\mathrm{al}^{7}$, the mean sensitivity to Penicillin and Ciprofloxacin were $70.83 \%$ and $60 \%$ respectively. Staphylococcus aureus showed $75 \%$ resistance to Methicillin, Oxacillin and Vancomycin. Uropathogens were sensitive to Norfloxacin, Cotrimoxazole and Ofloxacin. These results were in contrast to our study in which Escherchia coli was sensitive to Amoxycillin and Ciprofloxacin in only $6 \%$ and $15.6 \%$ respectively, and in case of Klebsiella pneumoniae none was sensitive to Amoxycillin and only $40 \%$ were sensitive to Ciprofloxacin. The reason for this difference may be due to the injudicious over the counter use of antibiotics.

In the study done by Mostafa et $\mathrm{al}^{8}$, Escherichia coli had a sensitivity rate of $97.8 \%$ to Ceftriaxone and $95.2 \%$ to Cefotaxime in contrast to our study in which Cefotaxim was sensitive only in $18.7 \%$ of cases. The highest resistance rate of Escherichia coli was to Penicillin (95.2\%) followed by Amoxycillin and Cotrimoxazole (79 and $74.2 \%$ respectively) in the study by Mostafa et al ${ }^{11}$ whereas in our study Escherichia coli showed high resistant to Amoxycillin (93\%) followed by Nalidixic acid (87.5\%). According to Mostafa et $\mathrm{al}^{8}$ Klebsiella spp. showed the highest sensitivity to Ciprofloxacin (95.1) and Ceftriaxone $90.7 \%$ which was in contrast to our study in which Klebsiella was sensitive to Ciprofloxacin and Cefotaxim only in $40 \%$ and $30 \%$ respectively. in our study Klebsiella showed highest resistant to Amoxycillin which was simiar to study done by Mostafa et $\mathrm{al}^{8}$.

In Study done by Rehamet $\mathrm{al}^{9}, 59.9 \%$ isolates of Escherichia coli were multidrug resistant where as in our study only one Escherichia coli was multidrug resistant. In our study Escherichia coli showed increase in resistant to oral antibiotics; Amoxycillin (93\%), Ofloxacin (81.2\%), Ciprofloxacin (84.3\%) Cotrimoxazole (68.75\%), and Nitrofurantoin $(46.8 \%)$ which was similar to the results in study done by Rehamet $\mathrm{al}^{9}$. The reason may be easy access to oral antibiotics from pharmacy.

In our study E. coli was sensitive to Cefotaxim in only $18.75 \%$ where as in study done by CW Kwan et $\mathrm{al}^{10}$, Escherichia coli was sensitive to greater than $95 \%$ of third-generation Cephalosporins (Ceftriaxone and Ceftazidime). In their study bacteria were frequently resistant to ampicillin (54.4\%) and TrimethoprimSulfamethoxazole $(40.4 \%)$ which was comparable to our study.

In our study Escherichia coli (81.2\%) and Klebsiella pneumoniae $(70 \%)$ showed increase in resistance pattern to Cephalosporins which was comparable to study done by Stephanie A et al ${ }^{14}$ in which there was high rate of resistance to third generation Cephalosporins in subpopulations of children admitted to the hospital for UTIs.

In the study done by Rasoulet al $^{15}$, most isolates showed high resistance against Ampicillin, Cotrimoxazole, Nalidixic acid, and Nitrofurantoin and Klebsiella isolates showed more resistance against tested antibiotics than Escherichia coli isolates which was comparable to our study.

Although our study suggested high resistance to oral antibiotics, there was a caveat that this study included in-patients only. This might have caused some selection bias. However, this information raises an important issue regarding antibiotic resistance in UTI. A future study including both in-patients and out-patients would help clarify if resistance to oral antibiotics has indeed emerged in the organisms causing UTI in the community.

\section{Conclusion}

Escherichia coli is the most common organism causing urinary tract infection in children. Urine microscopy as well as urine culture sensitivity should be sent before starting antibiotics. Uropathogens showed increased resistance to oral antibiotics. Amikacin remains the first line intravenous antibiotic in hospitalized patients. 


\section{References}

1. Supriya S. Tankhiwale, Suresh V. Jalgaonkar, Sarfraz Ahamad and Umesh Hassani. Evaluation of extended spectrum beta lactamase in urinary isolates. Indian J Med Res 2004;120:553-556.

2. Kahlmeter G. Eco.Sens. An international survey of the antimicrobial susceptibility of pathogens from uncomplicated urinary tract infections: the ECO.SENS Project. J Antimicrobial Chemother 2003;51:69-76.

3. Chakupurakal R, Ahmed M, Sobithadevi DN, Chinnappan S, Reynolds T. Urinary tract pathogens and resistance pattern. J Clin Pathol 2010;63:652-654.

4. Lutter SA, Currie ML, Mitz LB, Greenbaum LA. Antibiotic resistance patterns in children hospitalized for urinary tract infections. Arch Pediatr Adolesc Med 2005;159:924-928.

5. Arvind Bagga. Consensus Statement on Management of Urinary Tract Infections: Indian Pediatrics 2001;38:1106-1115.

6. I. Yolbas, R. Tekin1, S. Kelekci, A. Tekin, M.H. Okur, A. Ece, A. Gunes, V. Sen. Community-acquired urinary tract infections in children: pathogens, antibiotic susceptibility and seasonal changes. European Rev Med Pharmacol Sci 2013;17:971-976.

7. Durgesh D. Wasnik, P. M. Tumane. Prevalence and antibacterial susceptibility pattern of Urinary Tract Infection Causing Human Pathogenic Bacteria. Asian J Biomed Pharmaceutical Sci 2012;2:1-3.

8. Mostafa Sharifian, Abdollah Karimi, Sedigheh Rafiee, Tabatabaei and Navid Anvaripour. Microbial Sensitivity Pattern in Urinary Tract Infections in Children: A Single Centre Experience of 1,177 Urine Cultures. J Infect Dis 2006;59:380-382.
9. Rehamlssa Al-Mardeni, Adel Batarseh, Lina Omanish, Majdolin Shraideh, Basma Batarseh, Nidal Unis. Emprical Treatment for Pediatric Urinary Tract Infection and Resistance Patterns of Uropathogens, in Queen Alia Hospital and Prince A'Isha Military Centre - Jordan. Saudi J Kidney Dis Transplant 2009;20(1):135-139.

10. CW Kwan, $\mathrm{H}$ Onyett. Community-acquired urinary tract pathogens and their resistance patterns in hospitalized children in south-eastern Ontario between 2002 and 2006. Paediatr Child Health 2008;13:759-762.

11. Haller M, Brandis M, Berner R. Antibiotic resistance of urinary tract pathogens and rationale for empirical intravenous therapy. Pediatr Nephrol 2004;19:982986.

12. Prais $D$, Straussberg $R$, Avitzur $Y$, Nussinovitch $M$, Harel L, Amir J. Bacterial susceptibility to oral antibiotics in community acquired urinary tract infection. Arch Dis Child 2003;88:215-218.

13. Hoberman A, Wald ER, Hickey RW, et al. Febrile children oral versus initial intravenous therapy for urinary tract infections in young. Pediatrics. 1999;104;79-86.

14. Stephanie A. Lutter, MD; Melissa L. Currie, MD; Lindsay B. Mitz, BA; Larry A. Greenbaum, MD, PhD. Antibiotic Resistance Patterns in Children Hospitalized for Urinary Tract Infections. Arch Pediatr Adolesc Med. 2005;159:924-928.

15. Rasoul Yousefi Mashouf, Hooshang Babalhavaeji and Javad Yousef. Urinary Tract Infections: Bacteriology and Antibiotic Resistance Patterns. Indian Pediatrics. 2009;46: 617-620. 